\title{
Causes and Clinical Outcomes of Revision Total Knee Arthroplasty
}

\author{
Dong-Hyun Lee, MD, Seung-Hun Lee, MD, Eun-Kyoo Song, MD, Jong-Keun Seon, MD, Hong-An Lim, MD, \\ and Hong-Yeol Yang, MD \\ Department of Orthopedic Surgery, Center for Joint Disease, Chonnam National University Hwasun Hospital, Hwasun, Korea
}

\begin{abstract}
Purpose: The purpose of this study was to evaluate causes and clinical outcomes of revision total knee arthroplasty (TKA) with a minimum 2-year follow-up.

Materials and Methods: We performed a retrospective review on the records of 225 revision TKAs performed from 2003 to 2012 at a single institution. Finally, 206 cases were conducted. To evaluate clinical outcomes, we checked the range of motion (ROM), Knee Society (KS) score, Hospital for Special Surgery (HSS) score, and Western Ontario and McMaster Universities Osteoarthritis Index (WOMAC) score preoperatively, 6 months postoperatively and yearly thereafter and complications after revision TKA.

Results: The causes of revision TKA were septic complications in 120 and aseptic complications in 86 . The main aseptic complication was periprosthetic fracture in 36, followed by 25 loosening, 13 polyethylene wear and 9 instability. At the final follow-up, patients showed improvements in ROM and KS, HSS and WOMAC scores. Of the total, 7 patients underwent re-revision due to reinfection in 6 and periprosthetic fracture in 1.

Conclusions: Due to development of implants and surgical techniques, mechanical complications such as aseptic loosening and instability that need a revision decreased whereas septic complications relatively increased. Therefore, we should be cautious for the occurrence of infection. Overall, revision TKA provided clinical improvement in knee function and patient satisfaction.
\end{abstract}

Keywords: Knee, Arthroplasty, Revision, Cause, Outcome

\section{Introduction}

Total knee Arthroplasty (TKA) has been known to be significantly effective for patients with knee osteoarthritis and 95\% of the prostheses last at least 15 years ${ }^{1,2)}$. According to the latest worldwide joint registries, Sibanda et al. ${ }^{3)}$ reported that revision rates dropped due to the use of advanced implant designs, sophisticated surgical techniques and measures for infection

Received June 23, 2016; Revised (1st) September 4, 2016;

(2nd) October 16, 2016; Accepted December 10, 2016

Correspondence to: Eun-Kyoo Song, MD

Department of Orthopedic Surgery, Center for Joint Disease, Chonnam National University Hwasun Hospital, 322 Seoyang-ro, Hwasun-eup, Hwasun 58128, Korea

Tel: +82-61-379-7677, Fax: +82-62-379-7681

E-mail: eksong@chonnam.ac.kr

This is an Open Access article distributed under the terms of the Creative Commons Attribution Non-Commercial License (http://creativecommons.org/licenses/by-nc/4.0/) which permits unrestricted non-commercial use, distribution, and reproduction in any medium, provided the original work is properly cited. prevention. However, due to the greater need for primary TKA, the incidence of revision TKA has also increased ${ }^{3)}$. Hence, it is of high importance to assess postoperative outcomes of revision TKA.

Revision TKAs are performed early (within 2 to 5 years after primary surgery) in $60 \%-80 \%$ of the cases to extend the longevity of prostheses and achieve better clinical outcomes ${ }^{4)}$. However, revision TKA is indispensable in most cases: patients who undergo primary TKA eventually need revision TKA due to wear of machinery, aseptic loosening, infection, instability, malalignment and periprosthetic fractures ${ }^{2}$. Unfortunately, due to the difficulty of handling bone loss and soft tissue insufficiency during the unavoidable procedure, its outcomes are not as desirable as those of primary arthroplasty ${ }^{5-9)}$. Few studies have addressed postoperative complications of revision TKA and limited local data are available on revision TKA. The object of this study was to evaluate the causes and outcomes of revision TKA in a single institution with a minimum 2-year follow-up. 


\section{Materials and Methods}

This study was approved by the Institutional Review Board of our hospital. There was an increase in the incidence of revision TKA at our institution from 2003 to 2012. So, we retrospectively reviewed 225 cases (225 patients) of revision TKA performed by the same surgeon using both femoral and tibial prostheses at our institution from 2003 to 2012. Of the total 225 patients, 206 were included in this study excluding 19 patients who had been lost to follow-up. Clinical and radiological outcomes of revision TKA were assessed in these 206 patients. Their mean age at the time of revision surgery was $68.8 \pm 7.4$ years (range, 51 to 79 years). The mean interval from primary TKA to revision TKA was 73.0 \pm 6.2 months (range, 59 to 81 months) and the follow-up duration was $66.0 \pm 4.2$ months (range, 57 to 73 months).

Septic complications that necessitate revision TKA were diagnosed based on the criteria used to define infection. Patients presenting with infectious symptoms such as pain, swelling and burning sensation of the knee joint were evaluated for infection based on the following 4 criteria: 1) positive bacterial culture of the specimen from the first-stage procedure or more than 5 polymorphonuclear neutrophils per field; 2) a white blood cell count (WBC) of $\geq 15,000$ cells $/ \mathrm{mm}^{3}$ with hypersegmented neutrophils $\geq 90 \%$; 3 ) an erythrocyte sedimentation rate (ESR) of $\geq 70-80$ $\mathrm{mm} / \mathrm{hr}$ or a C-reactive protein (CRP) level of $10.0 \mathrm{mg} / \mathrm{dL}$; and 4 ) presence of draining fistulas ${ }^{10}$. If any of the above 4 criteria were satisfied, infection was diagnosed.

In the septic complication group, two-stage reimplantation was performed using mobile antibiotic impregnated cement spacers (Fig. 1). If an infection was diagnosed, implant removal and debridement were thoroughly performed in the operating room. The kind of antibiotics used for the cement spacer in the first stage procedure was decided as those sensitive to cultured organisms. In the cases with negative culture results, $2 \mathrm{~g}$ of vancomycin was mixed with $40 \mathrm{~g}$ bone cement containing $1 \mathrm{~g}$ erythromycin. In the cases with suspected fungal infection, $50 \mathrm{mg}$ of amphotericin was added. The cement spacer was created using each package of bone cement for the tibial area and femoral area. If the preoperative culture was positive, sensitive antibiotics were intravenously injected; if negative, $1 \mathrm{~g}$ of first generation cephalosporins was administered. The antibiotics were changed according to the intraoperative culture and then intravenous administration was continued for 6 weeks. The second-stage reimplantation was planned when systemic symptoms accompanying knee pain, burning sensation, open wounds and fistulas disappeared and laboratory findings such as WBC, ESR, and CRP were normal in more than two consecutive assessments performed with an interval of 1 month. The final revision procedure was performed if 1) less than 5 polymorphonuclear leukocytes were observed in the intraoperative frozen section biopsy of specimens taken from
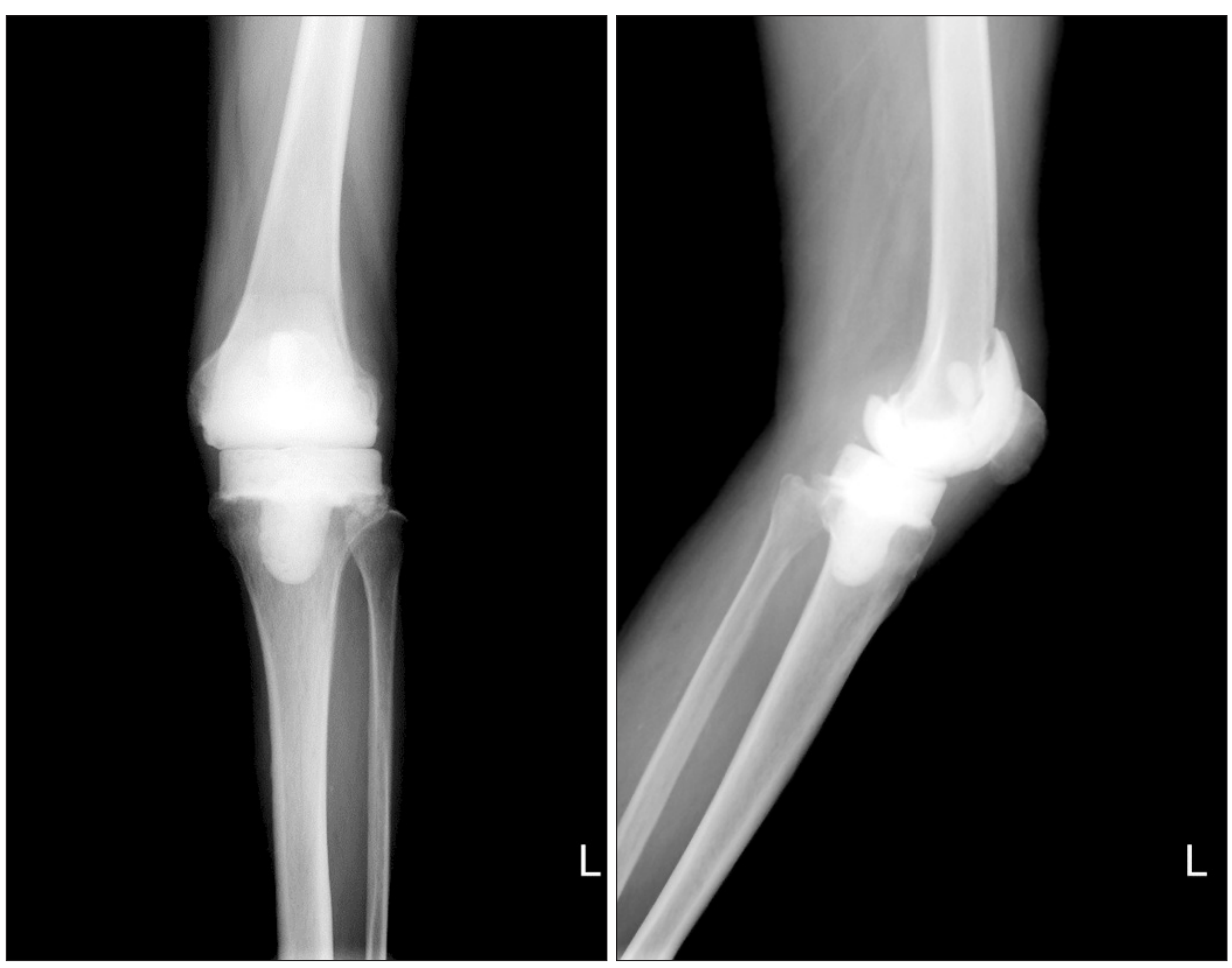

Fig. 1. Radiographs showing a mobile cement spacer. 
more than 3 areas with a magnification of $\times 400$ and 2) there was no gross evidence in intraoperative findings. In cases with 5-10 polymorphonuclear leukocytes, revision was determined if infection was considered resolved based on the assessment of clinical symptoms, postoperative condition, laboratory findings, and intraoperative naked-eye inspection.

We defined instability as abnormal and excessive displacement of the reticular elements that leads to clinical failure of the arthroplasty and one of the most common causes of aseptic failure following TKA ${ }^{11}$. All revision prostheses used in this study were the semi-constrained type, including Nexgen LCCK (Zimmer, Warsaw, IN, USA) in 155 knees and Triathlon (Stryker Orthopaedics, Mahwah, NJ, USA) in 51 knees. LCCK was used in 65 patients in the aseptic complication group and in 90 patients in the septic complication group whereas the Triathlon system was used in 21 patients in the aseptic complication group and in 30 patients in the septic complication group. We used a cementing technique for all implants. In revision TKA of the septic complication group, we used a full cementing technique using susceptible antibiotics whereas in the aseptic complication group, we used a partial cementing technique using susceptible antibiotics.

Postoperatively, continuous passive motion was initiated from the 1st postoperative day. Active motion exercises and quadriceps femoris strengthening exercises were started from the 2nd postoperative day. Once normal quadriceps femoris strength was achieved, indicating restoration of strength similar to that of the opposite leg, partial weight bearing with crutch assistance was initiated. In periprosthetic fracture cases, weigh bearing ambulation was not permitted until radiographic evidence of union was

Table 1. Demographic Data of Revision Total Knee Arthroplasty (TKA) Patients

\begin{tabular}{lc}
\hline \multicolumn{1}{c}{ Variable } & No. \\
\hline Gender & 34 \\
Male & 172 \\
Female & $68.8 \pm 7.4$ \\
Age (yr) & \\
Causes of primary TKA & 193 \\
Osteoarthritis & 12 \\
Rheumatoid arthritis & 1 \\
Charcot neuropathy & \\
Causes of revision TKA & 120 \\
Septic & 86 \\
Aseptic & $73.0 \pm 6.2$ \\
Interval of revision TKA (yr)
\end{tabular}

achieved. The patients were instructed to continue with the rehabilitation program after hospital discharge and asked for regular follow-ups at 3, 6, 9, and 12 months after surgery and once a year thereafter.

Clinical and radiographic assessments were performed in all patients preoperatively and at the final follow-up. The clinical outcomes were evaluated based on the range of motion (ROM), Hospital for Special Surgery (HSS) score, Knee Society (KS) score and Western Ontario and McMaster Universities Index (WOMAC) score. For the radiologic evaluation, radiolucency around the femur and tibia was assessed on the anteroposterior and lateral radiographs.

\section{Statistical Analysis}

According to normality test, intergroup comparisons were made using parametric Student $t$-test and the nonparametric Mann-Whitney $U$-test. Statistical analysis was done using IBM SPSS ver. 20.0 (IBM Co., Armonk, NY, USA). A p-value of 0.05 or less was considered to be statistically significant.

\section{Results}

There was an increase in the occurrence of revision TKA from 2003 to 2012. There were 34 males and 172 females in this study and all patients had primary TKA at our institution. Septic complications occurred in 120 patients (58.2\%) and aseptic complications in 86 patients $(41.8 \%)$ after primary TKA (Table 1$)$. The incidence of septic complications increased more rapidly than the aseptic ones (Fig. 2). Among these 206 revision TKA patients, 7 patients underwent re-revision surgery; 6 patients had reinfection; and 1 patient had a periprosthetic fracture.

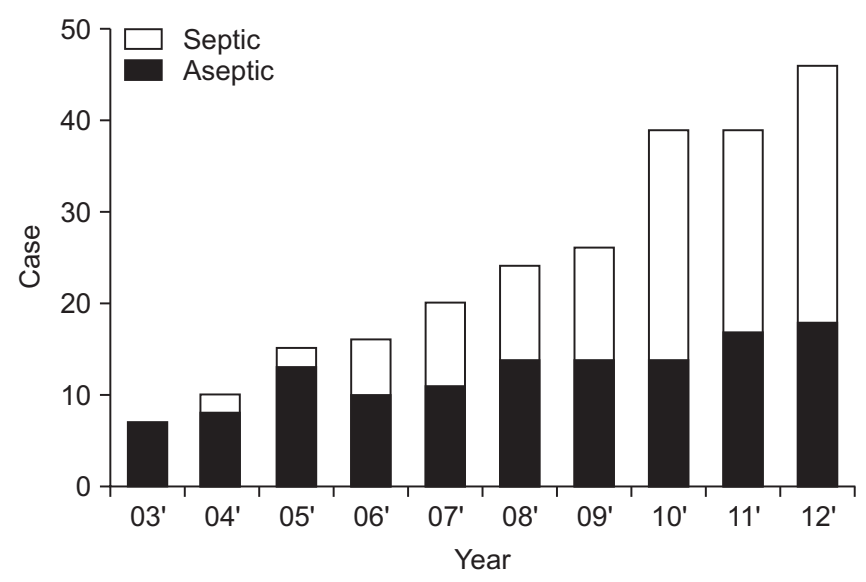

Fig. 2. From July 2003 to December 2012, a total of 225 revision total knee arthroplasties were performed at our institution. 
The main pathogens of infection were Staphylococcus aureus (25.7\%), Staphylococcus epidermidis (13.3\%), and Streptococcus (13.3\%). No organisms were found in 23 patients (25.7\%) (Table 2). The two-stage revision protocol was applied to all patients with periprosthetic infection.

Regarding the aseptic complications, there were periprosthetic fractures in 36 (41.9\%), aseptic loosening in 25 (29.1\%), polyethylene wear in $13(15.1 \%)$, instability in $9(10.5 \%)$, recurrent dislocation in $2(2.3 \%)$ and malalignment in 1 (1.1\%) (Table 3). All patients who underwent re-revision were included in the aseptic complication group. When evaluating cases with periprosthetic fractures, it was found that aseptic loosening had occurred ahead in most cases and such cases were classified as aseptic loosening when symptoms of pain or instability were observed before fracture. On the other hand, if there were no symptoms of pain or instability, they were diagnosed as periprosthetic fractures. In 13 cases with mechanical wear, component failure was categorized as mechanical wear but malalignment was not. There were 9 cases of instability without dislocation history leading to revision knee arthroplasty.

The average preoperative ROM for patients was $86.3^{\circ}\left(65.2^{\circ}\right.$ in

Table 2. Causative Organisms for Revision Total Knee Arthroplasty

\begin{tabular}{lc}
\hline \multicolumn{1}{c}{ Pathogen } & No. of knees (\%) \\
\hline Staphylococcus aureus & $29(24)$ \\
Staphylococcus epidermidis & $15(13)$ \\
Escherichia coli & $11(9)$ \\
Mycobacterium tuberculosis & $12(10)$ \\
Streptococcus & $15(13)$ \\
Staphylococcus hominis & $4(3)$ \\
Enterococcus & $1(1)$ \\
Enterobacter aerogenes & $4(3)$ \\
No organisms found & $29(24)$ \\
Total & $120(100)$ \\
\hline
\end{tabular}

Table 3. Aseptic Complications Requiring Revision Total Knee Arthroplasty

\begin{tabular}{lc}
\hline \multicolumn{1}{c}{ Complication } & No. of knees (\%) \\
\hline Periprosthetic fracture & $36(41.9)$ \\
Aseptic loosening & $25(29.1)$ \\
Polyethylene wear & $13(15.1)$ \\
Instability & $9(10.5)$ \\
Recurrent dislocation & $2(2.3)$ \\
Malalignment & $1(1.1)$ \\
Total & $86(100)$ \\
\hline
\end{tabular}

septic cases and $99.9^{\circ}$ in aseptic cases, $\left.\mathrm{p}=0.004\right)$, which increased to $111.4^{\circ}\left(101.7^{\circ}\right.$ in septic cases and $117.8^{\circ}$ in aseptic cases, $\mathrm{p}=$ 0.008 ) at the last follow-up, showing a statistically significant improvement $(\mathrm{p}<0.001)$ (Table 4$)$.

The preoperative KS score was 44.4 on average, which increased to 82.6 at the last follow-up, showing a statistically significant improvement $(\mathrm{p}<0.001)$. The difference between septic cases and aseptic cases was statistically significant postoperatively $(\mathrm{p}=0.017)$ (Table 5).

The HSS score demonstrated great improvement as well: preoperatively, the mean score was 52.5 , which increased to 82.5 at the last follow-up $(\mathrm{p}<0.001)$. A significant difference was seen between the two groups at the last follow-up $(\mathrm{p}=0.039)$ (Table 5).

The WOMAC score showed statistically significant improvement: the preoperative score was 60.8 on average, which decreased to 23.7 at the last follow-up ( $<<0.001$ ). There was also significant difference postoperatively between septic cases and aseptic cases $(\mathrm{p}=0.005)$ (Table 5).

On comparison of clinical outcomes according to the prosthesis used, there was no statistically significant difference.

\section{Complications}

Seven patients underwent re-revision TKA using LCCK in 2 years after revision TKA. Six of them had reinfection and 1 had a periprosthetic fracture at a mean of $24.0 \pm 2.2$ months after re-

Table 4. Range of Motion

\begin{tabular}{cccrc}
\hline Variable & Septic group & Aseptic group & \multicolumn{1}{c}{ Total } & p-value ${ }^{\text {a) }}$ \\
\hline Preoperative $\left(^{\circ}\right)$ & $65.2 \pm 42.0$ & $99.9 \pm 38.0$ & $86.3 \pm 48.0$ & 0.004 \\
Postoperative $\left(^{\circ}\right)$ & $101.7 \pm 17.2$ & $117.8 \pm 20.9$ & $111.4 \pm 18.9$ & 0.008 \\
\hline
\end{tabular}

${ }^{\mathrm{a})}$ Statistically significant $(\mathrm{p}<0.05)$.

Table 5. Comparison of Pre- and Postoperative Clinical Scores

\begin{tabular}{lcccc}
\hline Characteristic & Septic group & Aseptic group & Total & p-value \\
\hline HSS score & & & & \\
Preoperative & $47.4 \pm 14.2$ & $56.5 \pm 18.4$ & $52.5 \pm 19.7$ & 0.238 \\
Postoperative & $79.2 \pm 9.8$ & $85.5 \pm 10.2$ & $82.5 \pm 13.2$ & 0.039 \\
KS score & & & & \\
Preoperative & $44.1 \pm 17.9$ & $44.6 \pm 14.9$ & $44.4 \pm 16.7$ & 0.885 \\
Postoperative & $75.5 \pm 11.9$ & $86.6 \pm 9.6$ & $82.6 \pm 12.6$ & 0.017 \\
WOMAC score & & & & \\
Preoperative & $60.8 \pm 13.5$ & $60.9 \pm 14.1$ & $60.8 \pm 14.0$ & 0.468 \\
Postoperative & $30.3 \pm 13.9$ & $21.2 \pm 11.5$ & $23.7 \pm 15.5$ & 0.005 \\
\hline
\end{tabular}

HSS: Hospital for Special Surgery, KS: Knee Society, WOMAC: Western Ontario and McMaster Universities Osteoarthritis Index.

${ }^{a}$ Statistically significant $(\mathrm{p}<0.05)$. 
vision TKA. One knee was revised at 11 months after revision TKA for femoral shaft periprosthetic fracture. The patient was a 70-year-old woman with osteoporosis. The remaining 6 patients underwent implant removal and cement spacer insertion operation because of reinfection of the operated knee caused by the same previous pathogen, Staphylococcus aureus.

\section{Discussion}

Though TKA is known to be a highly effective procedure, revision TKA is a major challenge for surgeons and patients. Among surgeons, revision TKA has been associated with technical difficulty requiring a longer operation time and better management of blood loss than primary TKA ${ }^{7}$. For patients, it increases the risk of having other medical complications. Unfortunately, about $6 \%$ of those who undergo TKA need revision in 5 years $^{4}$. Even with continuous developments in surgical techniques and implant models, revision rates keep rising ${ }^{6}$. Furthermore, the rates will increase more drastically as primary TKA rates will increase in the future ${ }^{3)}$.

As shown in this study, septic complications mostly contributed to the increased revision rate. Development in surgical techniques may be related to the lower incidence of aseptic complications. In contrast, septic complications requiring a revision TKA continued to rise. This may be attributable to several factors. First, we cannot ignore the patient factors such as diabetes mellitus, smoking, malnutrition, and old age that should be managed strictly. Second, as the rate of aseptic complications decreases due to the development of implants, the rate of septic complications seems to increase relatively. Therefore, with the demand for primary TKA increasing, we need to pay more attention to infection that occur after primary TKA and patient factors by strictly controlling postoperative glucose levels, prohibiting smoking and shower before suture removal and managing diabetes mellitus and life style.

Regarding the failure of revision TKA, a significant number of patients (13 out of 19) had re-revision TKA due to reinfection. This is another indication that infection is a more critical complication than aseptic complications. Though there have been developments in antibiotics and knowledge of infection control, deeper understanding and more research on periprosthetic infection are necessary.

According to previous studies, the outcomes of revision TKA including the postoperative ROM may not be as successful in patients with infection as those in patients without infection ${ }^{12-18)}$. However, it has been recently proposed that using impregnated cement spacers to replenish for bone loss and soft tissue fibrosis could result in comparably successful outcomes in revision TKA for infection ${ }^{18,19)}$. Wang et al. ${ }^{20)}$ reported that while the noninfection revision TKA group obtained higher knee scores and greater ROM, the infection revision TKA group obtained the same function scores and patient satisfaction. Besides, according to Patil et al. ${ }^{21)}$, the infected group had even higher scores on Short Form 36 score, a quality of life index, and knee score. In the current study, the clinical outcomes based on the HSS score, KS score and WOMAC score were more satisfying in the aseptic complication group at statistically significant levels $(\mathrm{p}<0.05)$. The pre- and postoperative ROMs were notably greater in the aseptic complication group $(\mathrm{p}<0.05)$. We attributed these results to the fact that the ROM before the primary procedure was limited in the septic complication group, continuous operations resulted in more soft tissue and skin damage and the ROM was restricted due to the use of antibiotic impregnated cement spacers in the two-stage reimplantation.

Fehring et al. ${ }^{8)}$ analyzed the causes of revision in 279 patients: 105 patients (38\%) underwent revision for infection, 74 patients (27\%) for instability, 37 patients (13\%) for failure of ingrowth into porous components, 22 patients (8\%) for patellofemoral complications, 21 patients (7\%) for mechanical wear or osteolysis, 8 patients (3\%) for aseptic loosening and 12 patients for miscellaneous problems. Sharkey et al.9) reported a review on 212 revision knee arthroplasties. Among the reasons for revision, there were polyethylene wear (25\%), aseptic loosening (24.1\%), instability (21.2\%), infection (17.5\%), arthrofibrosis (14.6\%), malalignment (11.8\%), extensor mechanism deficiency (6.6\%), avascular necrosis of the patella (4.2\%) periprosthetic fracture $(2.8 \%)$ and isolated patella resurfacing $(0.9 \%)$.

There are some limitations to this study. First, the reviewed revision TKA data were from a single institution. In addition, the incidence of septic complications requiring revision TKA was high compared to that in other studies ${ }^{8,9}$, which may be associated with the relatively worse clinical data in the study.

Second, the wide range of follow-up period was another limitation. In patients with a short-term follow-up, the possibility of having complications that can occur in the long term, such as osteolysis and loosening, was overlooked. The results could be affected by prosthesis problems and clinical results do not represent the whole outcomes of revision TKA.

Third, we did not include radiological outcomes in the analysis considering that the use of different implants between the groups could hurt the accuracy of comparison. 


\section{Conclusions}

In conclusion, due to development of implants and surgical techniques, mechanical complications such as aseptic loosening and instability that need revision decreased; however, septic complications that require revision TKA relatively increased. Therefore, we should be cautious for the occurrence of infection after TKA. Overall, revision TKA showed clinical improvement in terms of knee function and patient satisfaction.

\section{Conflict of Interest}

No potential conflict of interest relevant to this article was reported.

\section{References}

1. Ranawat CS, Flynn WF Jr, Saddler S, Hansraj KK, Maynard MJ. Long-term results of the total condylar knee arthroplasty: a 15-year survivorship study. Clin Orthop Relat Res. 1993;(286):94-102.

2. Ritter MA, Berend ME, Meding JB, Keating EM, Faris PM, Crites BM. Long-term followup of anatomic graduated components posterior cruciate-retaining total knee replacement. Clin Orthop Relat Res. 2001;(388):51-7.

3. Sibanda N, Copley LP, Lewsey JD, Borroff M, Gregg P, MacGregor AJ, Pickford M, Porter M, Tucker K, van der Meulen JH; Steering Committee of the National Joint Registry (NJR) for England and Wales. Revision rates after primary hip and knee replacement in England between 2003 and 2006. PLoS Med. 2008;5:e179.

4. Labek G, Thaler M, Janda W, Agreiter M, Stockl B. Revision rates after total joint replacement: cumulative results from worldwide joint register datasets. J Bone Joint Surg Br. 2011; 93:293-7.

5. Hanssen AD, Rand JA, Osmon DR. Treatment of the infected total knee arthroplasty with insertion of another prosthesis: the effect of antibiotic-impregnated bone cement. Clin Orthop Relat Res. 1994;(309):44-55.

6. Bourne RB, Maloney WJ, Wright JG. An AOA critical issue: the outcome of the outcomes movement. J Bone Joint Surg Am. 2004;86:633-40.

7. Bozic KJ, Katz P, Cisternas M, Ono L, Ries MD, Showstack J. Hospital resource utilization for primary and revision total hip arthroplasty. J Bone Joint Surg Am. 2005;87:570-6.

8. Fehring TK, Odum S, Griffin WL, Mason JB, Nadaud M.
Early failures in total knee arthroplasty. Clin Orthop Relat Res. 2001;(392):315-8.

9. Sharkey PF, Hozack WJ, Rothman RH, Shastri S, Jacoby SM. Insall Award paper: why are total knee arthroplasties failing today? Clin Orthop Relat Res. 2002;(404):7-13.

10. Mortazavi SM, Molligan J, Austin MS, Purtill JJ, Hozack WJ, Parvizi J. Failure following revision total knee arthroplasty: infection is the major cause. Int Orthop. 2011;35:1157-64.

11. Song EK, Seon JK, Yim JH, Netravali NA, Bargar WL. Robotic-assisted TKA reduces postoperative alignment outliers and improves gap balance compared to conventional TKA. Clin Orthop Relat Res. 2013;471:118-26.

12. Bae DK, Yoon KH, Kim HS, Song SJ, Yi JW, Kim YC. The results of revision total knee arthroplasty. J Korean Orthop Assoc. 2003;38:689-94.

13. Goldberg VM, Figgie MP, Figgie HE 3rd, Sobel M. The results of revision total knee arthroplasty. Clin Orthop Relat Res. 1988;(226):86-92.

14. Windsor RE, Insall JN, Urs WK, Miller DV, Brause BD. Twostage reimplantation for the salvage of total knee arthroplasty complicated by infection: further follow-up and refinement of indications. J Bone Joint Surg Am. 1990;72:272-8.

15. Barrack RL, Engh G, Rorabeck C, Sawhney J, Woolfrey M. Patient satisfaction and outcome after septic versus aseptic revision total knee arthroplasty. J Arthroplasty. 2000;15:990-3.

16. Hwang SC, Cho SH, Jeong ST, Yune YP, Hwang IH. Clinical outcomes of infective and non-infective groups in revision total knee arthroplasty. J Korean Knee Soc. 2005;17:91-8.

17. Insall JN, Dethmers DA. Revision of total knee arthroplasty. Clin Orthop Relat Res. 1982;(170):123-30.

18. Goldstein WM, Kopplin M, Wall R, Berland K. Temporary articulating methylmethacrylate antibiotic spacer (TAMMAS): a new method of intraoperative manufacturing of a custom articulating spacer. J Bone Joint Surg Am. 2001;83 Suppl 2 Pt 2:92-7.

19. Hofmann AA, Goldberg T, Tanner AM, Kurtin SM. Treatment of infected total knee arthroplasty using an articulating spacer: 2- to 12-year experience. Clin Orthop Relat Res. 2005;(430):125-31.

20. Wang CJ, Hsieh MC, Huang TW, Wang JW, Chen HS, Liu CY. Clinical outcome and patient satisfaction in aseptic and septic revision total knee arthroplasty. Knee. 2004;11:45-9.

21. Patil N, Lee K, Huddleston JI, Harris AH, Goodman SB. Aseptic versus septic revision total knee arthroplasty: patient satisfaction, outcome and quality of life improvement. Knee. 2010;17:200-3. 